\title{
Formulae for the Values of Zeta and $L$-functions at Half Integers
}

\author{
Masao TOYOIZUMI \\ Rikkyo University \\ (Communicated by T. Mitsui)
}

\section{Introduction}

Ramanujan's formula for the Riemann zeta function at half integers was formulated and established by $Y$. Matsuoka [3]. And he deduced interesting representations for $\zeta(1 / 2) \zeta(2 a-1 / 2)$ and $\zeta(-1 / 2) \zeta(2 a+1 / 2)$, where $\zeta(s)$ is the Riemann zeta function and $a$ is an integer greater than 1. Recently, the author [4] generalized the results of Y. Matsuoka [3]. The purpose of this paper is to derive similar results for the values of zeta and $L$-functions.

\section{$\S 1$. Notations and results.}

Throughout this paper, we assume that $a$ is a positive integer and $b$ is a non-negative integer. As usual, $\boldsymbol{N}$ and $\boldsymbol{Q}$ denote the set of natural numbers and the field of rational numbers, respectively. Let $\chi$ be a primitive non-principal character modulo $k$ and $L(s, \chi)$ the Dirichlet $L$ function associated with $\chi$. Denote by $\bar{\chi}$ the character conjugate to $\chi$. Let $B_{n, \chi}$ denote the $n$-th Bernoulli number corresponding to $\chi$ in the sense of Leopoldt. For any positive integer $n$, we set

$$
g_{1}(a, b, \chi ; n)=\sum_{\substack{n_{1} n_{2} n_{3} \mid n \\\left(n_{1}, n_{2}, n_{3}\right) \in N^{3}}} \chi\left(n_{1}\right) \bar{\chi}\left(n_{2}\right) n_{1}^{-b-1 / 2} n_{2}^{2 a-1} n_{3}^{2 a-b-3 / 2}
$$

and

$$
g_{2}(a, b, \chi ; n)=\sum_{\substack{n_{1} n_{2} n_{3} \mid n \\\left(n_{1}, n_{2}, n_{3}\right) \in N^{3}}} \chi\left(n_{1}\right) \bar{\chi}\left(n_{2}\right) n_{1}^{-b-1 / 2} n_{2}^{2 a} n_{3}^{2 a-b-1 / 2}
$$

For brevity we will often write

$$
g_{i}(n)=g_{i}(a, b, \chi ; n) \quad(i=1,2) .
$$

Received April 8, 1980 
Further for any non-negative integer $m$, we put

and

$$
\varepsilon_{1}(m)=(-1)^{m(m+1) / 2}
$$

$$
\varepsilon_{2}(m)=(-1)^{m(m+3) / 2} \text {. }
$$

Then our formulae are formulated as follows.

TheOREM 1. Let $\chi$ be an even character. Assume $2 a \geqq b+1$, and define, for $x>0$,

$$
\begin{aligned}
G_{1}(a, b, \chi ; x) & \\
= & x^{2 a-b-1 / 2}\left\{\sum_{j=0}^{b} \sum_{n=1}^{\infty} \frac{2^{j}(2 b-j) ! g_{1}(n)}{2^{b}(b-j) ! j !}(4 \sqrt{n / k} x)^{j} e^{-4 \sqrt{n / k} x}\right. \\
& \left.+\frac{(-1)^{a-1} \varepsilon_{1}(b)(2 b) !(4 a-2 b-2) ! B_{2 a, \bar{\chi}} L\left(b+\frac{1}{2}, \chi\right) \zeta\left(2 a-b-\frac{1}{2}\right)}{a \cdot b !(2 a-b-1) ! 2^{b a-2 b-1} \pi^{2 a-b-1}}\right\} .
\end{aligned}
$$

Then for arbitrary positive numbers $\alpha, \beta$ with $\alpha \beta=\pi^{2}$, we have

$$
G_{1}(a, b, \chi ; \alpha)=G_{1}(a, b, \chi ; \beta) \text {. }
$$

Theorem 2. Let $\chi$ be an odd character. Assume $2 a \geqq b$, and define, for $x>0$,

$$
\begin{aligned}
G_{2}(a, b, \chi ; x) & \\
= & x^{2 a-b+1 / 2}\left\{\sum_{j=0}^{b} \sum_{n=1}^{\infty} \frac{2^{j}(2 b-j) ! g_{2}(n)}{2^{b}(b-j) ! j !}(4 \sqrt{n / k} x)^{j} e^{-4 \sqrt{n / k} x}\right. \\
& \left.+\frac{(-1)^{a-1} \varepsilon_{2}(b)(2 b) !(4 a-2 b) ! B_{2 a+1, \bar{x}} L\left(b+\frac{1}{2}, \chi\right) \zeta\left(2 a-b+\frac{1}{2}\right)}{(2 a+1) \cdot b !(2 a-b) ! 2^{b a-2 b+1} \pi^{2 a-b}}\right\} .
\end{aligned}
$$

Then for arbitrary positive numbers $\alpha, \beta$ with $\alpha \beta=\pi^{2}$, we have

$$
G_{2}(a, b, \chi ; \alpha)=G_{2}(a, b, \chi ; \beta) \text {. }
$$

For brevity, we put, for $x>0$,

$$
\begin{aligned}
& E_{i}(a, b, \chi ; x) \\
& \quad=\sum_{j=0}^{b} \sum_{n=1}^{\infty} \frac{2^{j}(2 b-j) ! g_{i}(n)}{2^{b}(b-j) ! j !}(\pi \sqrt{n / k} x)^{j} e^{-\pi \sqrt{n / k} x} \quad(i=1,2) .
\end{aligned}
$$

Then, by setting $(\alpha, \beta)=(2 \pi, \pi / 2)$ in (1), (2), we obtain the following 
corollaries.

Corollary 1. Let $a, b$ and $\chi$ be as in Theorem 1. Then we have

$$
\begin{aligned}
& L\left(b+\frac{1}{2}, \chi\right) \zeta\left(2 a-b-\frac{1}{2}\right) \\
& \quad=C_{1} \pi^{2 a-b-1}\left(E_{1}(a, b, \chi ; 2)-2^{4 a-2 b-1} E_{1}(a, b, \chi ; 8)\right),
\end{aligned}
$$

where

$$
C_{1}=\frac{(-1)^{a-1} \varepsilon_{1}(b) a \cdot b !(2 a-b-1) ! 2^{8 a-2 b-1}}{(2 b) !(4 a-2 b-2) !\left(2^{4 a-2 b-1}-1\right) B_{2 a, \bar{x}}}
$$

Corollary 2. Let $a, b$ and $\chi$ be as in Theorem 2. Then we have

$$
\begin{aligned}
& L\left(b+\frac{1}{2}, \chi\right) \zeta\left(2 a-b+\frac{1}{2}\right) \\
& \quad=C_{2} \pi^{2 a-b}\left(E_{2}(a, b, \chi ; 2)-2^{4 a-2 b+1} E_{2}(a, b, \chi ; 8)\right)
\end{aligned}
$$

where

$$
C_{2}=\frac{(-1)^{a-1} \varepsilon_{2}(b)(2 a+1) \cdot b !(2 a-b) ! 2^{8 a-2 b+1}}{(2 b) !(4 a-2 b) !\left(2^{4 a-2 b+1}-1\right) B_{2 a+1, \bar{x}}}
$$

Now, let $K$ be a quadratic field with the discriminant $d$ and $\chi_{d}=\left(\frac{d}{)}\right)$ the Kronecker symbol. Then it is well known that $\chi_{d}$ is a real primitive character modulo $|d|$ and

$$
L\left(s, \chi_{d}\right) \zeta(s)=\zeta_{K}(s),
$$

where $\zeta_{K}(s)$ is the Dedekind zeta function of $K$ (cf. [1]). Then from Corollaries 1 and 2 , we obtain the following results.

COROLLARY 3. Let $K$ be a real quadratic field, and assume $2 a \geqq$ $b+1$. Then we have

$$
\begin{aligned}
\zeta_{K}(b+ & \left.\frac{1}{2}\right) \zeta_{K}\left(2 a-b-\frac{1}{2}\right) \\
= & C_{3} \pi^{2 a-1}\left(E_{1}\left(a, b, \chi_{d} ; 2\right)-2^{4 a-2 b-1} E_{1}\left(a, b, \chi_{d} ; 8\right)\right) \\
& \times\left(E_{1}\left(a, 2 a-b-1, \chi_{d} ; 2\right)-2^{2 b+1} E_{1}\left(a, 2 a-b-1, \chi_{d} ; 8\right)\right),
\end{aligned}
$$

where

$$
C_{3}=\frac{(-1)^{a}}{\left(2^{4 a-2 b-1}-1\right)\left(2^{2 b+1}-1\right)}\left(\frac{a \cdot b !(2 a-b-1) ! 2^{4 a}}{(2 b) !(4 a-2 b-2) ! B_{2 a, x_{d}}}\right)^{2}
$$


COROLLARY 4. Let $K$ be an imaginary quadratic field, and assume $2 a \geqq b$. Then we have

$$
\begin{aligned}
\zeta_{K}(b+ & \left.\frac{1}{2}\right) \zeta_{K}\left(2 a-b+\frac{1}{2}\right) \\
= & C_{4} \pi^{2 a}\left(E_{2}\left(a, b, \chi_{d} ; 2\right)-2^{4 a-2 b+1} E_{2}\left(a, b, \chi_{d} ; 8\right)\right) \\
& \times\left(E_{2}\left(a, 2 a-b, \chi_{d} ; 2\right)-2^{2 b+1} E_{2}\left(a, 2 a-b, \chi_{d} ; 8\right)\right),
\end{aligned}
$$

where

$$
C_{4}=\frac{(-1)^{a+b}}{\left(2^{4 a-2 b+1}-1\right)\left(2^{2 b+1}-1\right)}\left(\frac{(2 a+1) b !(2 a-b) ! 2^{4 a+1}}{(2 b) !(4 a-2 b) ! B_{2 a+1, \chi_{d}}}\right)^{2} .
$$

Throughout the rest of this section, we assume that $K$ is an imaginary quadratic field. Setting $a=b$ in Corollary 2, we get the following corollary.

Corollary 5.

$$
\zeta_{K}\left(a+\frac{1}{2}\right)=C_{b} \pi^{a}\left(E_{2}\left(a, a, \chi_{d} ; 2\right)-2^{2 a+1} E_{2}\left(a, a, \chi_{d} ; 8\right)\right),
$$

where

$$
C_{5}=\frac{(-1)^{a-1} \varepsilon_{2}(a)(2 a+1)(a !)^{2} 2^{4 a+1}}{(2 a) !^{2}\left(2^{2 a+1}-1\right) B_{2 a+1, \chi_{d}}}
$$

From Corollaries 4 and 5, we get the following.

CoRollary 6. For any positive integer a, we have

$$
\begin{aligned}
\zeta_{K}\left(\frac{1}{2}\right)= & C_{6}\left(E_{2}\left(a, 0, \chi_{d} ; 2\right)-2^{4 a+1} E_{2}\left(a, 0, \chi_{d} ; 8\right)\right) \\
& \times\left(E_{2}\left(a, 2 a, \chi_{d} ; 2\right)-2 E_{2}\left(a, 2 a, \chi_{d} ; 8\right)\right) \\
& \div\left(E_{2}\left(2 a, 2 a, \chi_{d} ; 2\right)-2^{4 a+1} E_{2}\left(2 a, 2 a, \chi_{d} ; 8\right)\right),
\end{aligned}
$$

where

$$
C_{8}=-\frac{2(2 a+1)^{2} B_{4 a+1, x_{d}}}{(4 a+1) B_{2 a+1, x_{d}}^{2}}
$$

Setting $a=b$ in Theorem 2, we get the following theorem.

TheOREM 3. Define, for $x>0$, 


$$
\begin{aligned}
G\left(a, K_{d} ; x\right) & \\
= & x^{a+1 / 2}\left\{\sum_{j=0}^{a} \sum_{n=1}^{\infty} \frac{2^{j}(2 a-j) ! g(n)}{2^{a}(a-j) ! j !}(4 \sqrt{n / \mid d} \mid x)^{j} e^{-4 \sqrt{n /|d|} \mid x}\right. \\
& \left.+\frac{(-1)^{a-1} \varepsilon_{2}(a)(2 a) !^{2} B_{2 a+1, x_{d}} \zeta_{K}\left(a+\frac{1}{2}\right)}{(2 a+1)(a !)^{2} 2^{4 a+1} \pi^{a}}\right\}
\end{aligned}
$$

where

$$
g(n)=g_{2}\left(a, a, \chi_{d} ; n\right) .
$$

Then for arbitrary positive numbers $\alpha, \beta$ with $\alpha \beta=\pi^{2}$, we have

$$
G\left(a, K_{d} ; \alpha\right)=G\left(a, K_{d} ; \beta\right) .
$$

\section{§. Proof of Theorem 1.}

The method of proof is similar to that used in [3] and [4]. So it is sufficient to sketch the argument.

Suppose that $\chi$ is even, and put

$$
\begin{aligned}
& \varphi_{1}(a, b, \chi ; s) \\
& \quad=\frac{2^{b} \Gamma(s) \Gamma\left(s+b+\frac{1}{2}\right) \zeta(s) L\left(s+b+\frac{1}{2}, \chi\right) L(s-2 a+1, \bar{\chi}) \zeta\left(s-2 a+b+\frac{3}{2}\right)}{\pi^{1 / 2}\left(\frac{2 \pi}{\sqrt{k}}\right)^{2 s}} .
\end{aligned}
$$

Using the functional equations of the gamma, zeta and $L$-functions, we obtain

$$
\varphi_{1}(a, b, \chi ; s)=\varphi_{1}\left(a, b, \chi ; 2 a-b-\frac{1}{2}-s\right) .
$$

Now, we define, for $t>0$,

$$
F_{1}(a, b, \chi ; t)=\sum_{j=0}^{b} C_{b, j}\left\{\sum_{n=1}^{\infty} g_{1}(n)\left(4 \pi \sqrt{\overline{n t / k})^{j}} e^{-4 \pi \sqrt{n t / k}}\right\},\right.
$$

where

$$
C_{b, j}=\frac{2^{j}(2 b-j) !}{2^{b}(b-j) ! j !} .
$$

By the same way as in [4], we know that the series 


$$
t^{s-1} \sum_{n=1}^{\infty} g_{1}(n)(4 \pi \sqrt{n t / k})^{j} e^{-4 \pi \sqrt{n t / k}} \quad(0 \leqq j \leqq b)
$$

converges absolutely in $t>0$ and uniformly in any interval $\delta \leqq t<\infty$ with $\delta>0$. Thus we get

$$
\begin{aligned}
\int_{0}^{\infty} F_{1} & (a, b, \chi ; t) t^{s-1} d t \\
= & \sum_{j=0}^{b} C_{b, j}\left\{\sum_{n=1}^{\infty} g_{1}(n)(4 \pi \sqrt{n / k})^{j} \int_{0}^{\infty} t^{s+j / 2-1} e^{-4 \pi \sqrt{n t / k}} d t\right\} \\
= & 2\left(\frac{4 \pi}{\sqrt{k}}\right)^{-2 s} \sum_{j=0}^{b} C_{b, j} \Gamma(2 s+j) \sum_{n=1}^{\infty} n^{-s} g_{1}(n) .
\end{aligned}
$$

Since

$$
\left|g_{1}(n)\right| \leqq n^{4 a+1}
$$

it follows that

$$
\sum_{n=1}^{\infty} n^{-8} g_{1}(n)=\zeta(s) L\left(s+b+\frac{1}{2}, \chi\right) L(s-2 a+1, \bar{\chi}) \zeta\left(s-2 a+b+\frac{3}{2}\right)
$$

for $\operatorname{Re}(s)>4 a+2$, and so for all $s$ (by the theorem of identity). Thus we get, from (5), (6) and the proposition of [4],

$$
\varphi_{1}(a, b, \chi ; s)=\int_{0}^{\infty} F_{1}(a, b, \chi ; t) t^{s-1} d t
$$

Since $\varphi_{1}(a, b, \chi ; s)$ is regular in $\operatorname{Re}(s)>2 a$, Mellin's inversion formula permits us to write

$$
F_{1}(a, b, \chi ; t)=\frac{1}{2 \pi i} \int_{2 a+1 / 2-i \infty}^{2 a+1 / 2+i \infty} \varphi_{1}(a, b, \chi ; s) t^{-s} d s
$$

Then it is easily verified that

$$
\begin{aligned}
& F_{1}(a, b, \chi ; t)=\frac{1}{2 \pi i} \int_{-(b+1)-i \infty}^{-(b+1)+i \infty} \varphi_{1}(a, b, \chi ; s) t^{-s} d s \\
& \quad+\left\{\text { sum of residues of integrand at poles } s=0,2 a-b-\frac{1}{2}\right\} .
\end{aligned}
$$

Substituting $s=2 a-b-1 / 2-S$, it follows from (4) and (7) that

$$
\frac{1}{2 \pi i} \int_{-(b+1)-i \infty}^{-(b+1)+i \infty} \varphi_{1}(a, b, \chi ; s) t^{-\bullet} d s=t^{-2 a+b+1 / 2} F_{1}\left(a, b, \chi ; \frac{1}{t}\right) \text {. }
$$

The residues in the sum are as follows: 


$$
\begin{aligned}
\operatorname{Res}_{s=0}\left(\varphi_{1}(a, b, \chi ; s) t^{-s}\right) & \\
= & \frac{(-1)^{a} \varepsilon_{1}(b)(2 b) !(4 a-2 b-2) ! B_{2 a, \bar{\chi}} L\left(b+\frac{1}{2}, \chi\right) \zeta\left(2 a-b-\frac{1}{2}\right)}{a \cdot b !(2 a-b-1) ! 2^{8 a-2 b-1} \pi^{2 a-b-1}} .
\end{aligned}
$$

$\underset{s=2 a-b-1 / 2}{\operatorname{Res}}\left(\varphi_{1}(a, b, \chi ; s) t^{-s}\right)$

$$
=\frac{(-1)^{a-1} \varepsilon_{1}(b)(2 b) !(4 a-2 b-2) ! B_{2 a, \bar{\chi}} L\left(b+\frac{1}{2}, \chi\right) \zeta\left(2 a-b-\frac{1}{2}\right) t^{-2 a+b+1 / 2}}{a \cdot b !(2 a-b-1) ! 2^{b a-2 b-1} \pi^{2 a-b-1}} .
$$

Using (8) and (9), these calculations give the equality

$$
\begin{aligned}
& F_{1}(a, b, \chi ; t)+(-1)^{a-1} A_{1} \\
& \quad=t^{-2 a+b+1 / 2}\left(F_{1}\left(a, b, \chi ; \frac{1}{t}\right)+(-1)^{a-1} A_{1}\right),
\end{aligned}
$$

where

$$
A_{1}=\frac{\varepsilon_{1}(b)(2 b) !(4 a-2 b-2) ! B_{2 a, \bar{\chi}} L\left(b+\frac{1}{2}, \chi\right) \zeta\left(2 a-b-\frac{1}{2}\right)}{a \cdot b !(2 a-b-1) ! 2^{6 a-2 b-1} \pi^{2 a-b-1}} .
$$

Setting $t=(\alpha / \pi)^{2}$ and $1 / t=(\beta / \pi)^{2}$, the above equality immediately yields Theorem 1.

\section{§3. Proof of Theorem 2.}

Suppose that $\chi$ is odd, and put

$$
\begin{aligned}
\varphi_{2}(a, b, \chi ; s) \\
=\frac{2^{b} \Gamma(s) \Gamma\left(s+b+\frac{1}{2}\right) \zeta(s) L\left(s+b+\frac{1}{2}, \chi\right) L(s-2 a, \bar{\chi}) \zeta\left(s-2 a+b+\frac{1}{2}\right)}{\pi^{1 / 2}\left(\frac{2 \pi}{\sqrt{k}}\right)^{2 s}} .
\end{aligned}
$$

Then we obtain

$$
\varphi_{2}(a, b, \chi ; s)=\varphi_{2}\left(a, b, \chi ; 2 a-b+\frac{1}{2}-s\right) .
$$

Next, we difine, for $t>0$,

$$
F_{2}(a, b, \chi ; t)=\sum_{j=0}^{b} C_{b, j}\left\{\sum_{n=1}^{\infty} g_{2}(n)(4 \pi \sqrt{n t / k})^{j} e^{-4 \pi \sqrt{n t / k}}\right\},
$$


where

$$
C_{b, j}=\frac{2^{j}(2 b-j) !}{2^{b}(b-j) ! j !} .
$$

By the same argument as in the proof of Theorem 1, we see that

$$
F_{2}(a, b, \chi ; t)=\frac{1}{2 \pi i} \int_{2 a+1-i \infty}^{2 a+1+i \infty} \varphi_{2}(a, b, \chi ; s) t^{-s} d s \text {. }
$$

Then it is easily verified that

$$
\begin{aligned}
& F_{2}(a, b, \chi ; t)=\frac{1}{2 \pi i} \int_{-(b+1 / 2)-i \infty}^{-(b+1 / 2)+i \infty} \varphi_{2}(a, b, \chi ; s) t^{-s} d s \\
& \quad+\left\{\text { sum of residues of integrand at poles } s=0,2 a-b+\frac{1}{2}\right\} .
\end{aligned}
$$

Substituting $s=2 a-b+1 / 2-S$, it follows from (10) and (11) that

$$
\frac{1}{2 \pi i} \int_{-(b+1 / 2)-i \infty}^{-(b+1 / 2)+i \infty} \varphi_{2}(a, b, \chi ; s) t^{-s} d s=t^{-2 a+b-1 / 2} F_{2}\left(a, b, \chi ; \frac{1}{t}\right) \text {. }
$$

The residues in the sum are as follows:

$$
\begin{aligned}
\operatorname{Res}_{s=0}\left(\varphi_{2}(a, b, \chi ; s) t^{-s}\right) & \\
= & \frac{(-1)^{a} \varepsilon_{2}(b)(2 b) !(4 a-2 b) ! B_{2 a+1, \bar{\chi}} L\left(b+\frac{1}{2}, \chi\right) \zeta\left(2 a-b+\frac{1}{2}\right)}{(2 a+1) \cdot b !(2 a-b) ! 2^{6 a-2 b+1} \pi^{2 a-b}} .
\end{aligned}
$$

$\underset{s=2 a-b+1 / 2}{\operatorname{Res}}\left(\varphi_{2}(a, b, \chi ; s) t^{-s}\right)$

$$
=\frac{(-1)^{a-1} \varepsilon_{2}(b)(2 b) !(4 a-2 b) ! B_{2 a+1, \bar{\chi}} L\left(b+\frac{1}{2}, \chi\right) \zeta\left(2 a-b+\frac{1}{2}\right) t^{-2 a+b-1 / 2}}{(2 a+1) \cdot b !(2 a-b) ! 2^{8 a-2 b+1} \pi^{2 a-b}} .
$$

Using (12) and (13), these calculations give the equality

$$
\begin{aligned}
& F_{2}(a, b, \chi ; t)+(-1)^{a-1} A_{2} \\
& \quad=t^{-2 a+b-1 / 2}\left(F_{2}\left(a, b, \chi ; \frac{1}{t}\right)+(-1)^{a-1} A_{2}\right),
\end{aligned}
$$

where

$$
A_{2}=\frac{\varepsilon_{2}(b)(2 b) !(4 a-2 b) ! B_{2 a+1, \bar{\chi}} L\left(b+\frac{1}{2}, \chi\right) \zeta\left(2 a-b+\frac{1}{2}\right)}{(2 a+1) \cdot b !(2 a-b) ! 2^{8 a-2 b+1} \pi^{2 a-b}} .
$$


Setting $t=(\alpha / \pi)^{2}$ and $1 / t=(\beta / \pi)^{2}$ in the above equality, we can easily deduce Theorem 2.

\section{References}

[1] R. Ayoub, An introduction to the analytic theory of numbers, Mathematical Surveys, No. 10, Amer. Math. Soc., 1963.

[2] K. Iwasawa, Lectures on $p$-adic $L$-functions, Princeton University Press, Princeton, 1972.

[3] Y. MatsuoKa, On the values of the Riemann zeta function at half integers, Tokyo J. Math., 2 (1979), 371-377.

[4] M. Toyorzumi, Formulae for the Riemann zeta function at half integers, Tokyo J. Math., 3 (1980), 177-186.

Present Address:

Department of Mathematics

RIKKYO UNIVERSITY

Nishi-IKEBUKURo, ToKyo 171 